\title{
FAST scanning in the developing world emergency department
}

\author{
Zoë A Smith, Naas Postma, Darryl Wood
}

Objectives. To assess the utility of an existing ultrasound machine for the purposes of focused assessment sonography in trauma (FAST) scanning in a developing world emergency department (ED).

Design. Prospective study undertaken over a 12-month period. Trauma patients attending the ED were FAST scanned by one of three trained emergency medicine doctors.

Setting. The ED at a government hospital in rural KwaZuluNatal (KZN), the referral centre for 22 peripheral hospitals.

Subjects. All patients presenting to the ED who had sustained abdominal or thoracic trauma.

Outcome measures. Scans were recorded as positive or negative for free intra-abdominal or pericardial fluid. All results were confirmed by computed tomography, laparotomy or a second trained ED ultrasonographer, followed by a period of clinical observation.
Results. 72 FAST scans were included, 52 for blunt trauma and 20 for penetrating trauma. Of the 72 scans, $15(20.8 \%)$ were positive. FAST scanning had $100 \%$ specificity and overall sensitivity of $71.4 \%$. When considering blunt trauma alone the sensitivity improved to $81.3 \%$, while in penetrating trauma it was much poorer $(62.5 \%)$

Conclusions. We propose a valuable role for FAST scanning in all peripheral hospitals for the assessment of patients sustaining blunt trauma. In rural areas with limited resources FAST scans may assist in the appropriate timely transfer of trauma patients for further imaging or definitive surgical intervention

S Afr Med J 2010; 100: 105-108.
Ngwelezane Hospital is a 554-bed government hospital situated in rural KwaZulu-Natal (KZN). It is the referral centre for 22 peripheral hospitals covering north-eastern $\mathrm{KZN}$ as far as $300 \mathrm{~km}$ away on the Mozambique border, and encompassing an estimated population of 3 million people. It has a 24-hour emergency department (ED) with a functional ultrasound scanner, computed tomography (CT) scanning capabilities, facilities for definitive surgical care, and an eight-bed intensive care unit. Most of these specialised services are not available in the majority of the rural district hospitals in this province. These tend to be resource poor and severely understaffed with poor access to radiology, particularly after hours.

South African EDs experience one of the highest trauma loads in the world. In 1999 the South African Medical Research Council estimated that 1.5 million trauma cases presented to secondary and tertiary hospitals alone. ${ }^{1}$ Treatment of trauma patients presents the emergency doctor and surgeon with significant diagnostic and therapeutic challenges. Developing mechanisms to reduce time to definitive care is therefore a priority in trauma management. ${ }^{2}$ Diagnostic peritoneal

Ngwelezane Hospital Emergency Department, KwaZulu-Natal Zoë A Smith, MB BCh, BSc, DTM\&H

Naas Postma, MB BCh, DipPEC

Darryl Wood, MB BCh, BPharm, MPhil, FCEM (UK), DipPEC, DA lavage (DPL) and CT scanning assist in correctly identifying patients who require early surgical intervention. More recently, emergency ultrasound has emerged in South African EDs as a novel diagnostic tool.

Focused assessment with sonography for trauma (FAST) is a rapid bedside ultrasound examination that was first developed in Europe and subsequently employed in other trauma units. ${ }^{3}$ FAST scanning is a portable sonographic technique performed by surgeons and ED doctors to screen for significant haemoperitoneum or pericardial tamponade after trauma. Free fluid may be detected in the pericardium and dependent areas in the abdominal cavity in a supine patient. Bleeding is usually the cause in the trauma context.

Several studies have investigated the reliability and accuracy of FAST scanning in trauma. A Cochrane systematic review found that the sensitivity for detecting haemoperitoneum in trauma patients was $85-95 \%$ and the specificity higher. ${ }^{4}$ FAST scanning expedites disposition of trauma patients, decreasing time to definitive care and reducing demands for CT scanning. ${ }^{5}$

We are not aware of studies investigating the use of FAST in a developing world setting. While emergency doctors working in tertiary centres in developed countries may have the luxury of choice between various diagnostic modalities, in most rural settings in South Africa CT scanning and radiology are not available after hours. However, decisions to transfer patients for definitive care must be made in an equally timely manner. We aimed to assess the use and accuracy of an existing ultrasound machine by recently trained ED doctors for the 


\section{Original Articles}

purposes of FAST scanning in our department. Our intention was to propose its wider use in peripheral hospitals.

\section{Methods}

This prospective study was undertaken from 1 January to 31 December 2008. FAST scans were performed on patients presenting to the Ngwelezane ED with suspected blunt or penetrating abdominal or thoracic trauma, as part of the American College of Surgeons Advanced Trauma Life Support (ATLS) $)^{2}$ secondary survey by three ED doctors accredited for FAST. Training involved attendance at either the emergency ultrasonography course provided by the American College of Emergency Physicians or the British College of Emergency Medicine emergency ultrasound course. All three doctors were required to perform a number of supervised scans before accreditation.

The sample of patients meeting the inclusion criteria was limited to periods when FAST-qualified doctors were present in the resuscitation unit. No exclusion from the study was made on the basis of sex, age or haemodynamic stability. Scans were performed on the supine patient with a pre-existing Aloka SSD 500 B-scan ultrasound machine (Aloka, Japan) with a $3.5 \mathrm{~Hz}$ abdominal probe. Right upper quadrant, left upper quadrant, pericardial and pelvic views were obtained as per FAST scanning principles. Results were documented as either positive or negative for free peritoneal or pericardial fluid (Figs 1 and 2).

FAST scan findings were subsequently supported by either CT scanning or laparotomy. Where this was not indicated findings were verified by a second qualified ED ultrasonographer repeating the FAST scan. Scans that were not confirmed by either of these techniques were excluded. All cases were documented with indication for scan, result, and final method of confirmation with any discrepancies in findings. Data were anonymised and analysed using Excel 2007.

\section{Results}

Over the 12-month study period, 91 FAST scans were performed in patients sustaining abdominal or thoracic trauma; 19 were excluded owing to failure to satisfactorily confirm scan results (17) or equivocal findings (2).

\section{All FAST scans $(N=72)$}

Of the 72 cases meeting the inclusion criteria, 52 scans were

for blunt trauma and 20 for penetrating trauma. Three patients died from their injuries before further imaging or surgical intervention; 2 had negative FAST scans and 1 was positive. Scans were confirmed by CT in 31 cases $(43.1 \%)$ and laparotomy in 17 (23.6\%). The remaining 24 (33.3\%) cases were rescanned by a second qualified ED ultrasonographer and observed clinically.

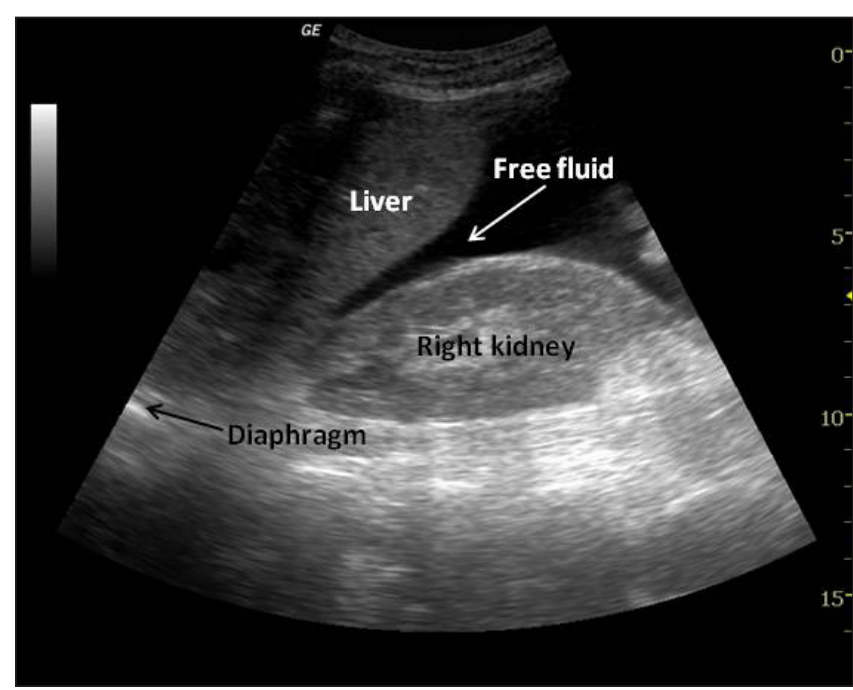

Fig. 1. Right upper quadrant view depicting a positive FAST scan obtained during the study period with free fluid visible in Morrison's pouch.

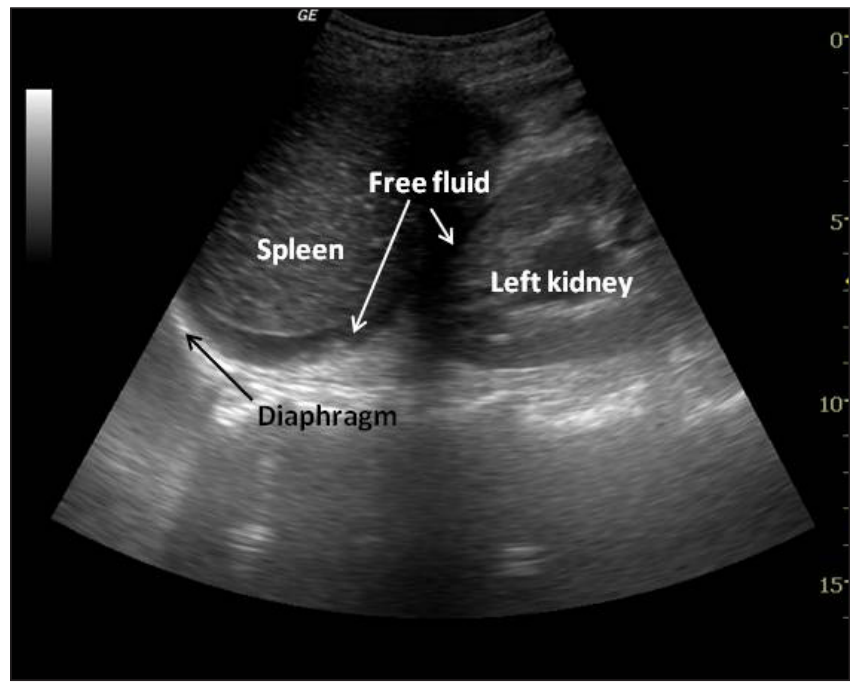

Fig. 2. Left upper quadrant view of the same patient showing free fluid in the splenorenal recess.

All the positive FAST scans (15 of 72) were confirmed by either CT or a positive laparotomy. FAST scanning therefore had $100 \%$ specificity in this study. Six of the remaining 57 scans $(10.5 \%)$ reported as negative were subsequently found to be positive. The overall sensitivity of all FAST scans was $71.4 \%$.

\section{Blunt trauma $(N=52)$}

The mechanism of injury was documented in $67.3 \%$ of blunt trauma cases. Of these, $85.7 \%$ involved a motor vehicle accident (in one-third of cases the patient was a pedestrian). Punitive assault by members of the community accounted for a further $14.3 \%$ of cases.

There were 13 positive and 39 negative FAST scans in blunt trauma. Three $(7.7 \%)$ were false negatives, of which 2 had $<500 \mathrm{ml}$ free fluid in the abdominal cavity on CT and $1 \mathrm{had}$ a ruptured bladder revealed at laparotomy. In blunt trauma 
alone, the sensitivity was $81.3 \%$ and negative predictive value $91.6 \%$.

\section{Penetrating trauma $(N=20)$}

There were $12 \mathrm{stab}$ wounds and 8 gunshot wounds. There were 5 positive and 15 negative FAST scans. The false-negative rate was $20.0 \%$ ( 3 of 15 cases). In all these cases injury to a solid viscus including retroperitoneal organs was found at laparotomy. Sensitivity in penetrating trauma was poor $(62.5 \%)$.

\section{Discussion}

Owing to the high specificity of FAST, international research has led to its being regarded as a good 'rule-in' tool for intraabdominal and intrathoracic trauma. In blunt trauma studies investigating FAST scanning, outcomes have demonstrated an average specificity of $90-99 \%$ and sensitivity of 86 $99 \% .^{4-8}$ Sensitivity in this study was therefore comparable. In accordance with previous evidence, our study also demonstrated excellent specificity of FAST $(100 \%)$ regardless of mechanism of injury.

Repeated scanning significantly increases the sensitivity of FAST. An increase from $78 \%$ with early FAST scans to above $90 \%$ was shown following repeated examinations for free intraabdominal fluid. ${ }^{9}$ Our study only included one FAST scan per patient, so it seems likely that the sensitivity might have been improved by serial scans in the ED.

Our study showed a higher rate of FAST positives (20.8\%) compared with other studies. In a comparable study in the UK, only 8 of 153 (5.2\%) scans for trauma were positive. This reflects the severity of injuries seen in this population in South Africa, and the challenges it represents.

FAST is often considered less sensitive than other methods of determining the extent of intra-abdominal injury such as DPL and CT scanning. The Cochrane review showed that the use of FAST in the treatment algorithm for blunt trauma did not have a significant effect on the final outcome of patients. ${ }^{4}$ However, a direct comparison of FAST and DPL showed FAST scans to be a good alternative, with a similar specificity and a much lower complication rate. ${ }^{10}$ FAST has since largely supplanted DPL for blunt trauma assessment. ${ }^{11}$ While CT scanning remains the gold standard in terms of radiological assessment, it has been proposed that FAST may be an acceptable alternative in resource-poor facilities, where CT is largely unavailable without transfer. ${ }^{12}$

FAST was only used to determine the presence of free intraperitoneal fluid or pericardial fluid, and not specific organ pathology. Despite the high negative predictive value $(91.6 \%)$ of FAST in blunt trauma in this study it is important to note that the absence of free fluid does not exclude serious intra-abdominal injury, and FAST should therefore be used as a 'rule-in' tool. Sonographic detection of visceral injury has low sensitivity, with one study revealing the presence of visceral injury on CT in $34 \%$ of patients with no evidence of haemoperitoneum. ${ }^{13}$ Organ injuries seen at laparotomy also account for the much poorer sensitivity of FAST $(62.5 \%)$ in penetrating trauma in our study. Specificity remained $100 \%$. These findings are in agreement with previous ${ }^{14}$ studies and indicate a less useful role of FAST scanning in penetrating trauma unless findings are positive.

In our setting, peripheral hospitals with no CT scanning and limited radiology could make use of ultrasound as a tool for supplying useful information to surgeons in referral hospitals. Trauma patients are referred to our ED for surgical opinion and interventions. Transfer times are up to 6 hours from the furthest outlying hospitals. Early ultrasound detection of free fluid in the trauma patient could be used as an indication to expedite transfer and reduce morbidity and mortality. This may involve decisions regarding mode of transportation and enlisting the services of aeromedical transport (Fig. 3). All 22 peripheral hospitals in our region have access to an ultrasound machine routinely used for obstetrics that could also be used for FAST.

\section{Limitations}

Limitations of this study include the use of an old and outdated ultrasound machine that affected picture quality. The lack of a functional printing or saving facility did not

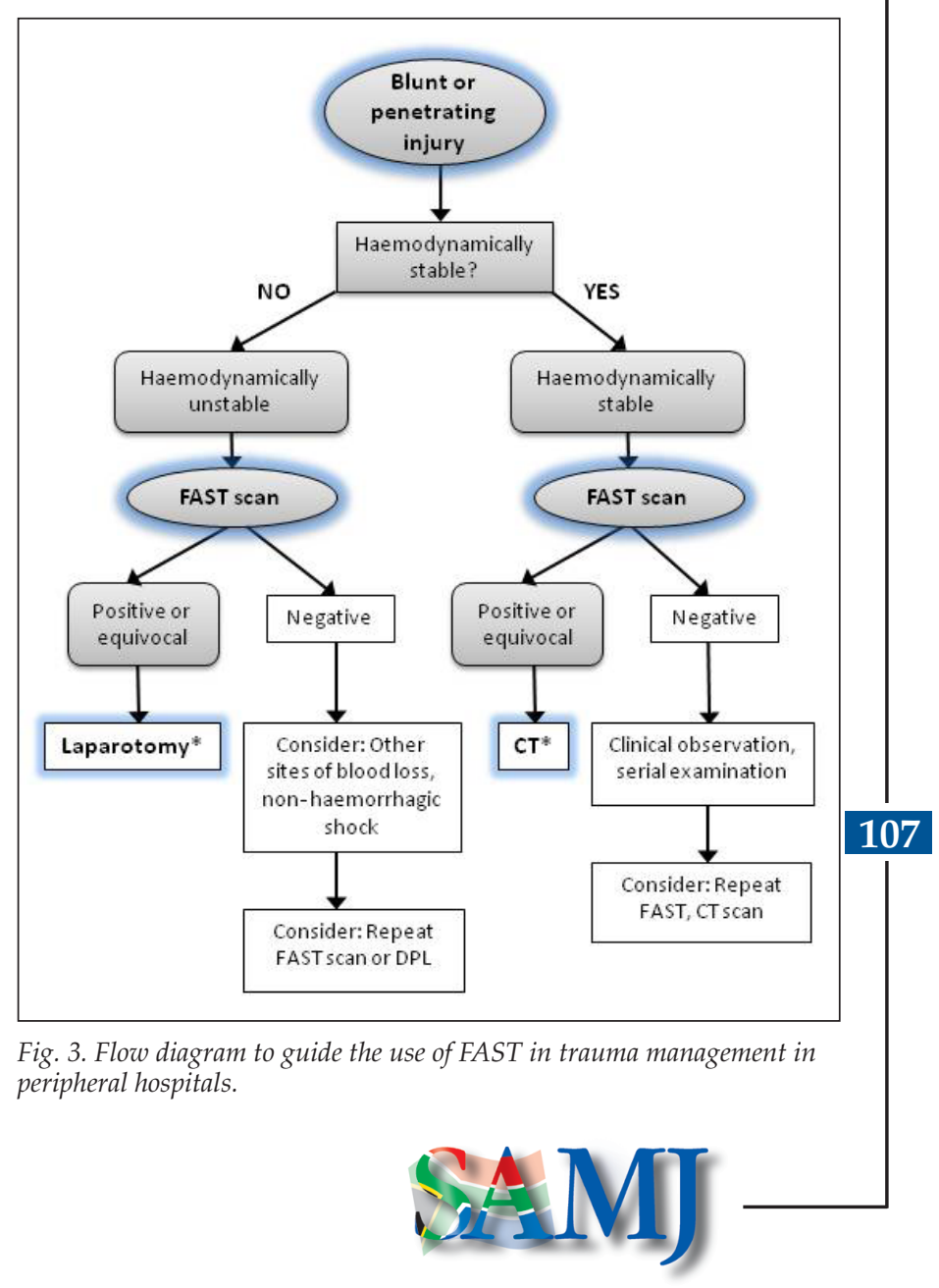




\section{Original Articles}

permit retrospective analysis of scans by a blinded observer. The second ultrasonographer was also not universally blinded to the results of the first ultrasonographer. FAST scans were limited to times when trained doctors were present in the resuscitation unit.

\section{Conclusions}

On the basis of our encouraging results we propose the use of FAST scanning in all peripheral hospitals to assess blunt trauma patients. In rural hospitals with limited resources and no facilities for advanced imaging or definitive surgical care, it can play a valuable role in primary and subsequent assessment and the timely transfer of appropriate trauma patients. Since most of these hospitals have ultrasound machines, implementation would be cheap, cost-effective and sustainable. There is a need for outreach programmes to train emergency doctors in FAST scanning in rural areas, and a South African accreditation system to standardise practice.

Conflicting interests. None.
References

Matzopoulos RG, Prinsloo MR, Butchart A, et al. Estimating the South African trauma caseload. Int I Inj Contr Saf Promot 2006; 13: 49-51.

2. Committee on Trauma. Advanced Trauma Life Support Provider's Manual. 7th ed. Chicago: American College of Surgeons, 2005.

. Noble VE, Nelson B, Sutingco AN. Manual of Emergency and Critical Care Ultrasound. New York: Cambridge University Press, 2007: 37-40.

4. Stengel D, Bauwens K, Sehouli J, et al. Emergency ultrasound-based algorithms for diagnosing blunt abdominal trauma. Cochrane Database Syst Rev 2005; 2: CD004446.

5. Melniker L, Liebner E, McKinney M, et al. Randomized clinical trial of point-of-care, limited ultrasonography for trauma in the emergency department: Sonography Outcomes Assessment Program (SOAP)-1 Trial. Ann Emerg Med 2004; $44(4$ suppl): S2

6. McKenney MG, Martin L, Lentz K, et al. 1,000 consecutive ultrasounds for blunt abdominal trauma. J Trauma 1996; 40: 607-612

7. Dolich MO, McKenney MG, Varela JE, et al. 2,576 ultrasounds for blunt abdominal trauma. J Trauma 2001; 50: 108-112

. Rozycki GS, Ballard RB, Feliciano DV, et al. Surgeon-performed ultrasound for the assessment of truncal injuries: lessons learned from 1540 patients. Ann Surg 1998; 228: 557-567.

9. Brenchley J, Walker A, Sloan JP, et al. Evaluation of focussed assessment with sonography in Trauma (FAST) by UK emergency physicians. Emerg Med J 2006; 23: 446-448.

10. Chambers JA, Pilbrow WJ. Ultrasound in abdominal trauma: an alternative to peritoneal lavage. Emerg Med J 1988; 5: 26-33.

11. Geeraedts L, Kaasjager H, Van Vugt A, et al. Exsanguination in trauma: A review of diagnostics and treatment options. Injury 2009; 40: 11-20.

2. Bakker J, Genders R, Mali W, et al. Sonography as the primary screening method in evaluating blunt abdominal trauma. J Clin Ultrasound 2005; 33: 155-163.

13. Shanmuganathan $\mathrm{K}$, Mirvis $\mathrm{SD}$, Sherbourne $\mathrm{CD}$, et al. Haemoperitoneum as the sole indicato of abdominal visceral injuries: a potential limitation of screening abdominal US for trauma. Radiology 1999; 212: 422-430

14. Udobi KF, Rodriguez A, Chiu WC. Role of ultrasonography in penetrating abdominal trauma: a prospective clinical study. J Trauma 2001; 50: 475-479. 